ARTICLE

Received 27 Jan 2015 | Accepted 27 May 2015 | Published 2 Jul 2015

DOI: $10.1038 /$ ncomms 8645

OPEN

\title{
Crystallographic structure of a small molecule SIRT1 activator-enzyme complex
}

Han Dai ${ }^{1,2}$, April W. Case ${ }^{1}$, Thomas V. Riera1 ${ }^{1}$ Thomas Considine ${ }^{1}$, Jessica E. Lee ${ }^{3}$, Yoshitomo Hamuro ${ }^{3}$, Huizhen Zhao², Yong Jiang², Sharon M. Sweitzer ${ }^{2}$, Beth Pietrak², Benjamin Schwartz², Charles A. Blum', Jeremy S. Disch1, Richard Caldwell', Bruce Szczepankiewicz¹, Christopher Oalmann', Pui Yee Ng1, Brian H. White ${ }^{1}$, Rebecca Casaubon ${ }^{1}$, Radha Narayan ${ }^{1}$, Karsten Koppetsch', Francis Bourbonais ${ }^{1}$, Bo Wu ${ }^{4}$, Junfeng Wang ${ }^{4}$, Dongming Qian ${ }^{5}$, Fan Jiang ${ }^{5}$, Cheney Mao ${ }^{5}$, Minghui Wang ${ }^{2}$, Erding $\mathrm{Hu}^{2}$, Joe C. Wu', Robert B. Perni ${ }^{1}$, George P. Vlasuk ${ }^{1} \&$ James L. Ellis ${ }^{1,2}$

SIRT1, the founding member of the mammalian family of seven NAD ${ }^{+}$-dependent sirtuins, is composed of 747 amino acids forming a catalytic domain and extended $\mathrm{N}$ - and C-terminal regions. We report the design and characterization of an engineered human SIRT1 construct (mini-hSIRT1) containing the minimal structural elements required for lysine deacetylation and catalytic activation by small molecule sirtuin-activating compounds (STACs). Using this construct, we solved the crystal structure of a mini-hSIRT1-STAC complex, which revealed the STAC-binding site within the N-terminal domain of hSIRT1. Together with hydrogendeuterium exchange mass spectrometry (HDX-MS) and site-directed mutagenesis using full-length hSIRT1, these data establish a specific STAC-binding site and identify key intermolecular interactions with hSIRT1. The determination of the interface governing the binding of STACs with human SIRT1 facilitates greater understanding of STAC activation of this enzyme, which holds significant promise as a therapeutic target for multiple human diseases.

\footnotetext{
${ }^{1}$ Sirtris, a GlaxoSmithKline Company, 200 Technology Square, Suite 300, Cambridge, Massachusetts 02139, USA. ${ }^{2}$ GlaxoSmithKline, 1250S. Collegeville Road, Collegeville, Pennsylvania 19426, USA. ${ }^{3}$ ExSAR Corporation, 11 Deer Park Drive, Suite 103, Monmouth Junction, New Jersey 08852, USA. ${ }^{4}$ High Magnetic Field Laboratory, Hefei Institutes of Physical Science, Chinese Academy of Sciences, 350 Shushanhu Road, Hefei, Anhui Province 230031, China. ${ }^{5}$ Viva Biotech, 334 Aidisheng Road, Zhangjiang High-tech Park, Shanghai 201203, China. Correspondence and requests for materials should be addressed to H.D. (email: Han.x.Dai@gsk.com).
} 
S rtuins are a family of highly conserved $\mathrm{NAD}^{+}$-dependent deacylases that have been linked to a number of important biological processes across a broad span of diverse organisms such as Saccharomyces cerevisiae, Caenorhabditis elegans, Drosophilla melanogaster and Mus musculus, among others $^{1,2}$. Sirtuins generally catalyze the deacylation of modified lysine residues in protein substrates coupled with the breakdown of $\mathrm{NAD}^{+}$into nicotinamide (NAM) and 2'-O-acyl-ADP-ribose. Of the seven sirtuins (SIRT1-7) that have been identified in mammals ${ }^{3}$, human SIRT1 (hSIRT1) is the most studied isoform, and has been shown to be regulated by calorie restriction and to be involved in multiple biological processes ${ }^{4-7}$. The validated, protective role of increased mammalian SIRT1 activity in metabolic disorders ${ }^{8}$, neurodegeneration ${ }^{9}$ and inflammation ${ }^{10,11}$ makes this enzyme an attractive therapeutic target. To this end, the development of pharmacological approaches to increase the enzymatic activity of hSIRT1 might lead to a new generation of therapeutic agents for a wide spectrum of diseases associated with aging. Small molecule sirtuin-activating compounds (STAC) have been developed which increase the catalytic deacetylation of specific Lys residues by hSIRT1 in multiple substrates, resulting in a variety of biological responses ${ }^{12-14}$. However, the molecular mechanism of hSIRT1 activation by STACs remains controversial. Questions as to whether STACs directly activate hSIRT1 persist ${ }^{15}$ despite evidence of allosteric activation ${ }^{13}$. Recently, a single point mutation of the $\mathrm{Glu}^{230}$ residue of hSIRT1 has been shown to attenuate kinetic activation by STACs ${ }^{16}$, further demonstrating a direct effect on hSIRT1. Structural characterizations of hSIRT1 fragments have shed light on the inhibitor binding and key regulatory element ${ }^{17,18}$. Similar to other sirtuins, hSIRT1 catalytic domain contains a Rossmann-fold large lobe and a zinc-binding small lobe and undergoes a significant conformational change of domain closure upon substrate/ligand occupying the active site ${ }^{19-21}$. However, the molecular details governing the binding of STACs to SIRT1 remain elusive, due to the difficulty in obtaining a detailed X-ray crystallographic structure of the full-length enzyme. To address this, we developed an engineered hSIRT1 (mini-hSIRT1) that is biochemically equivalent to the full-length enzyme with respect to basal catalytic activity and activation by STACs. X-ray crystallographic analysis of mini-hSIRT1 resulted in the first detailed structural determination of a fully functional human SIRT1 with a bound small molecule activator. The details of STAC binding to mini-hSIRT1 were translated to the full-length enzyme using structure-guided mutagenesis which corroborated the importance of key amino acids in the binding of STACs. These data are important in elucidating the molecular basis for STAC-mediated activation of hSIRT1 which will be critical for the development of future therapeutic agents.

\section{Results}

Mini-hSIRT1 design and characterization. To identify and characterize the key functional regions of hSIRT1, we performed hydrogen-deuterium exchange mass spectrometry (HDX-MS) on the full-length hSIRT1 protein. The rate of H-D exchange is highly dependent on the dynamic properties of the protein, with faster exchange occurring at solvent exposed and/or flexible regions and slower exchange occurring at the more buried and/or rigid regions ${ }^{22}$. Consistent with the previous study on hSIRT1(19-747) ${ }^{16}$, full-length hSIRT1 contains three major structured regions: the catalytic core region; residues 229-516 (referred to as hSIRT1cd hereafter) ${ }^{3,20}$; the N-terminal region of 183-229 immediately preceding the catalytic core and a remote region following the catalytic core around 641-665, previously reported as human $\mathrm{C}$-terminal regulatory segment (CTR) peptide $^{18}$; and murine essential for SIRT1 activity peptide ${ }^{23}$. (Fig. 1a and Supplementary Fig. 1a).

To probe the STAC-binding site on hSIRT1, HDX-MS was performed in the absence or presence of STAC 1 (Supplementary Fig. 2). Addition of 1 reduces the $H-D$ exchange rate around residues 183-229 in the N-terminal region of hSIRT1, suggesting that this domain is involved in STAC binding. Hereafter, this domain is referred to as the STAC-binding domain (SBD) (Fig. 1a,b and Supplementary Fig. 1a). Addition of 1 to hSIRT1 in the presence of a $\mathrm{p} 53$-derived peptide substrate $(\text { Ac-p53(W5) })^{13}$ results in perturbation of the $\mathrm{H}-\mathrm{D}$ exchange rates around the SBD and further protection at the substrate-binding site (residues 417-424) in the catalytic domain compared with the hSIRT1/Acp53(W5) complex, indicating that STAC binding in the $\mathrm{N}$-terminal domain and substrate binding within the catalytic domain of hSIRT1 are coupled (Fig. 1b). This is consistent with the previous observation that STACs enhance substrate binding to hSIRT1, thereby increasing hSIRT1 catalytic efficiency ${ }^{12}$.

In the absence of the CTR peptide, the catalytic core (hSIRT1cd) only shows $\sim 15 \%$ of the activity of the full-length enzyme using deacetylation assay conditions previously reported (Supplementary Fig. 1b) ${ }^{13}$. The addition of the CTR peptide restores the catalytic activity of hSIRT1cd, to $80 \%$ of that of fulllength hSIRT1, consistent with previous observations ${ }^{23,24}$ (Supplementary Fig. 1b). Kinetic characterization reveals that the CTR peptide restores activity by lowering the $K_{\mathrm{M}}$ values for both peptide substrate and $\mathrm{NAD}^{+}$of hSIRT1cd by $4-5$-fold (Supplementary Table 1).

Taken together, the above data suggest a tripartite architecture for a minimally functional hSIRT1 that includes: (1) the central domain constituting the basic catalytic machinery; (2) the $\mathrm{N}$-terminal SBD that mediates STAC binding and activation; and (3) the C-terminal CTR peptide which stabilizes the catalytic domain resulting in more efficient deacetylase activity. Based on this, we designed several hSIRT1 constructs encompassing all three of the minimal structural elements covalently bound, which we termed mini-hSIRT1s. The constructs span $183-505$ or 183516, which are connected to the CTR peptide via a flexible polyglycine/serine linker (GS, (GGGS) ${ }_{2}$ or $\left.(\mathrm{GGGS})_{3}\right)(\text { Fig. 1a })^{25}$. The $K_{\mathrm{M}}$ and $k_{\text {cat }}$ values are comparable between mini-hSIRT1 constructs and the full-length enzyme, as are the $\mathrm{IC}_{50}$ values for the hSIRT1 inhibitors EX-527 (ref. 26) or NAM, confirming functional fidelity of mini-hSIRT1s (Supplementary Tables 2 and 3 ). In addition, there is an excellent correlation between minihSIRT1 and the full-length enzyme with respect to STACmediated activation across a broad set of chemotypes (Fig. 1c). Removal of the SBD completely abolishes STAC-mediated activation of mini-hSIRT1, confirming the critical importance of this domain for activation (Fig. 1d). In contrast, mini-hSIRT1 lacking the CTR retains a significant level of STAC activation (Fig. 1e) suggesting that CTR is not required for STAC-mediated activation. Finally, the E230K mutation also attenuates STACmediated activation in mini-hSIRT1 as in the full-length enzyme $^{16}$ (Fig. 1f). Collectively, these observations demonstrate that at half the molecular size, mini-hSIRT1 can serve as an active and activatable surrogate for full-length hSIRT1.

Structure of the mini-hSIRT1-STAC complex. Although the $\mathrm{X}$-ray crystallographic structures of the hSIRT1 catalytic domain and complex of SIRT1cd/CTR have been reported ${ }^{17,18}$, no structure of the full-length enzyme is available. Solving the structure of the full-length hSIRT1 has been challenging, likely due to the conformational flexibility of the extended $\mathrm{N}$ - and C-terminal domains ${ }^{16,27}$. The mini-hSIRT1 constructs, which contain only the functionally critical regions of the $\mathrm{N}$ and 
a
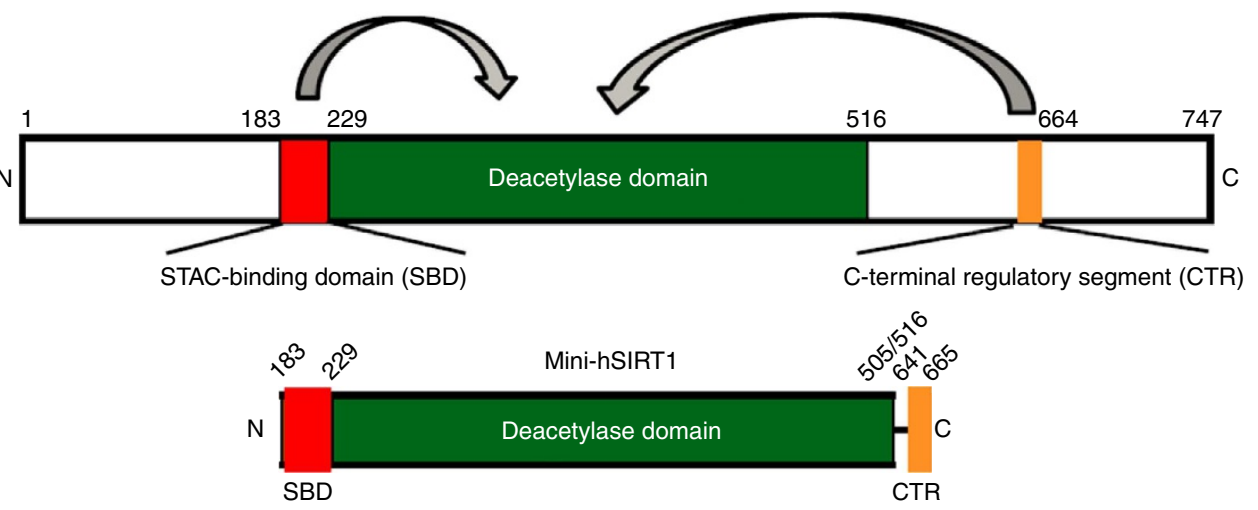

- GS, $(\mathrm{GGGS})_{2}$ or $(\mathrm{GGGS})_{3}$
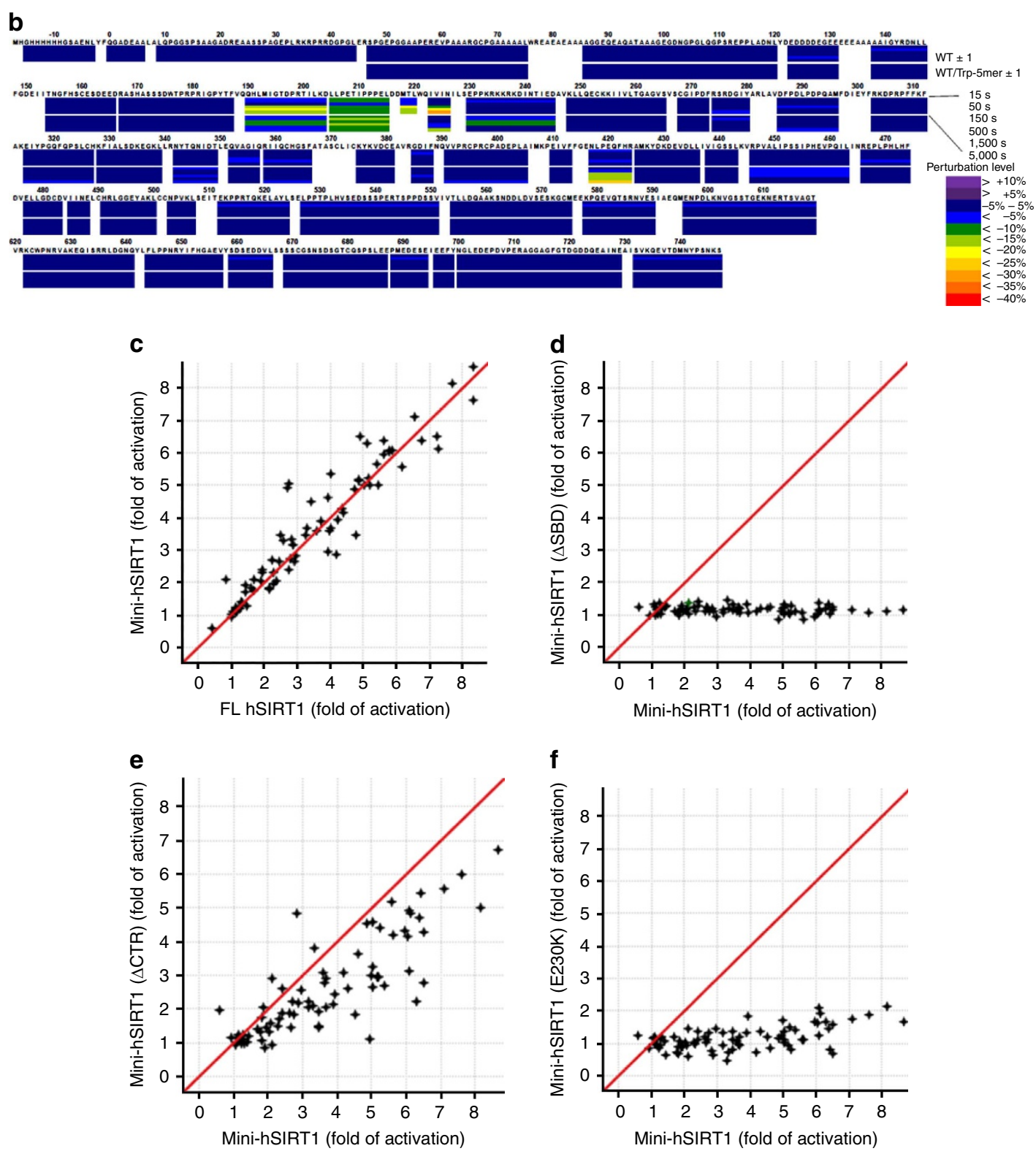

Figure 1 | Mini-hSIRT1 construct design and characterization. (a) Schematic diagram of human full-length hSIRT1 and Mini-hSIRT1 constructs. The $\mathrm{N}$-terminal SBD, the central catalytic domain and the CTR are highlighted in red, green and orange. (b) Heat map of the HDX-MS perturbation of binding of 1 to hSIRT1 in the absence or presence of Ac-p53(W5) (Trp-5mer) at six different time points (15-5000 s). (c) Pivot plot of the activation by a chemically diverse STAC set using the Ac-p53(W5) substrate for mini-hSIRT1 versus full-length hSIRT1, as measured by OAcADPR assay. The red line represents $y=x$ correlation. (d) Pivot plot of the STAC activation of mini-hSIRT1( $\triangle \mathrm{SBD}$ ) versus mini-hSIRT1. (e) Pivot plot the STAC activation of mini-hSIRT1( $\triangle C T R$ ) versus mini-hSIRT1. (f) Pivot plot of the STAC activation of mini-hSIRT1(E230K) versus mini-hSIRT1. 
C-terminal domains, afforded us the opportunity to crystallize a functional surrogate of the full-length enzyme. We successfully crystallized mini-hSIRT1 (183-505-(GGGS)2-CTR) with STAC 1 used in the HDX-MS experiments and determined the structure of the complex (mini-hSIRT1/1) at $3.1 \AA$ by molecular replacement using a search model based on the homologous model of SIRT3 (ref. 20).

Mini-hSIRT1 comprises a catalytic domain that assumes a Rossmann-fold large lobe and a zinc-binding small lobe common to all sirtuins ${ }^{19}$, an $\mathrm{N}$-terminal three-helical bundle encompassing the SBD and a C-terminal $\beta$-hairpin CTR peptide ${ }^{18}$ (Fig. 2a). The CTR peptide mediates $\beta$-augmentation with the six-stranded $\beta$-sheet of the Rossmann-fold lobe of the catalytic domain
(Fig. 2a), in agreement with the HDX-MS results of hSIRT1cd perturbation upon CTR binding (Supplementary Fig. 3a,b). The CTR-mediated $\beta$-augmentation appears to stabilize the active site of the hSIRT1cd which restores the $K_{\mathrm{M}}$ values observed for both acetylated peptide and $\mathrm{NAD}^{+}$substrates $^{18}$. The N-terminal SBD forms an independently folded, three-helix bundle with 1 binding to the helix-turn-helix (H2-T-H3) motif within the SBD, consistent with the HDX-MS and enzyme kinetic results (Fig. 2a). STAC 1 interacts extensively with the hydrophobic side chains of $\mathrm{Leu}^{206}, \mathrm{Thr}^{209}$ (methyl), Pro ${ }^{211}, \mathrm{Pro}^{212}$, $\mathrm{Leu}^{215}$, $\mathrm{Thr}^{219}$ (methyl), Ile ${ }^{223}$ and Ile ${ }^{227}$ from the H2-T-H3 motif with only one hydrophilic interaction: a hydrogen bond with $\mathrm{Asn}^{226}$ (Fig. 2b). The major mini-hSIRT1/1-binding site is a shallow
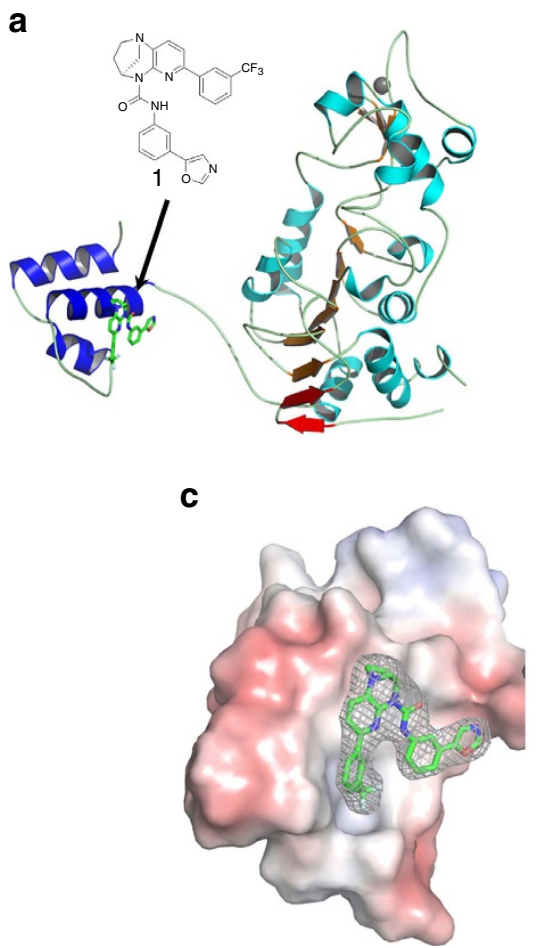

d

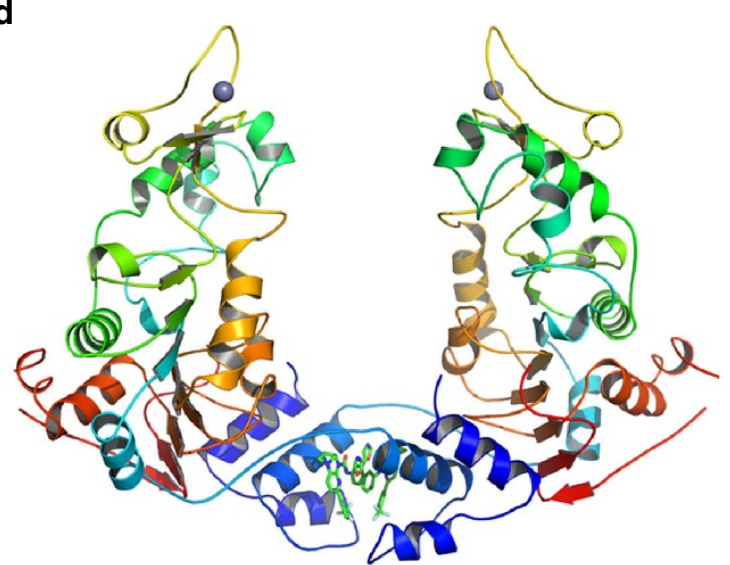

b
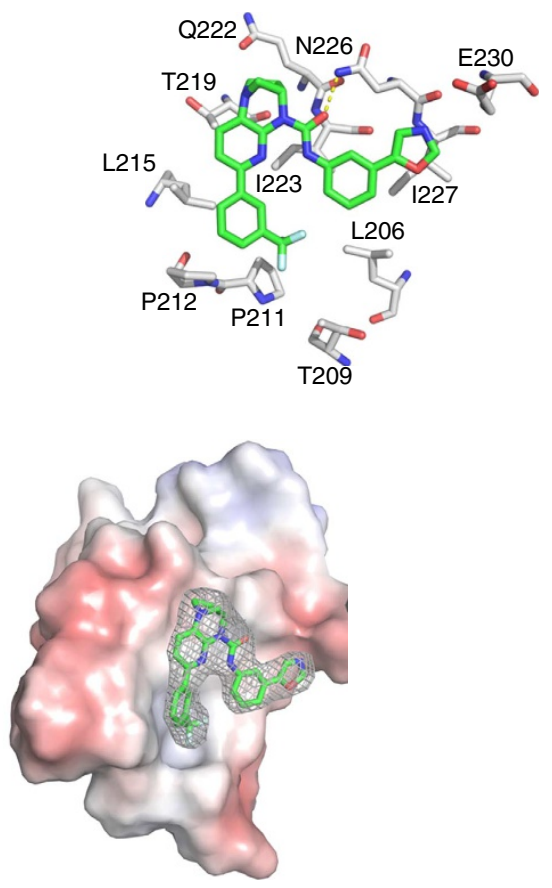
hydrophobic surface depression with an off-center, deeper hydrophobic pocket, that the $\mathrm{CF}_{3}$ group of $\mathbf{1}$ occupies (Fig. 2c). This is consistent with the observed structure-activity relationships developed across multiple STAC chemotypes, indicating the requirement of overall flatness of the core scaffold maintained by an intramolecular hydrogen bond ${ }^{28}$. A remarkable similarity in terms of domain configuration is observed between the mini-hSIRT1 structure and that of yeast Sir2 with both having an $\mathrm{N}$-terminal helical bundle and the C-terminal $\beta$-augmentation by a $\beta$-hairpin beyond the typical Rossmann-fold large lobe ${ }^{29}$ (Supplementary Fig. 3c). However, yeast Sir2 does not include the 130 amino acid insertion (510-640) observed in hSIRT1 and appears to be a natural 'mini-SIRT1' in yeast.

Interestingly, a STAC-mediated dimer of mini-hSIRT1 related by crystallographic symmetry was observed in the crystal lattices (Fig. 2d). Size exclusion chromatography (SEC) indicates that the apparent size of mini-hSIRT1 increases in the presence of STAC $\mathbf{1}$, which is likely correspondent to the mini-hSIRT1 dimer species (Supplementary Fig. 4). We are currently attempting to determine if the observed crystallographic dimer has any relevance in the observed biology of STAC-mediated SIRT1 activation.

In addition to the mini-hSIRT1/1 complex structure, we also determined a $2.73 \AA$ structure of a quaternary complex of minihSIRT1, 1, a seven amino acid peptide substrate derived from p53 (Ac-p53), and the nonhydrolyzable $\mathrm{NAD}^{+}$analog carbaNAD and a $2.74 \AA$ structure mini-hSIRT1/1 in complex with an activesite directed inhibitor 2 (ref. 30) that occupies the peptide and $\mathrm{NAD}^{+}$-binding sites (Fig. 3a,b and Supplementary Fig. 2). In the quaternary complex structure, the Ac-p53 peptide and carbaNAD bind to the active site cleft between the large and small lobes. Ac-p53 adopts an extended conformation, similar to the backbone-mediated $\beta$-strand like interactions observed in many Sirtuin/Ac-peptide complexes ${ }^{19,31}$. The main chain amide groups of Ac-p53 form hydrogen bonds with those of the residues $\mathrm{Gly}^{415}$ and $\mathrm{Glu}^{416}$ from the small lobe and those of the residues $\mathrm{Lys}^{444}$ and $\mathrm{Arg}^{446}$ from the large lobe (Supplementary Fig. 5a). The hydrogen bonds between the amide of the peptide +1 position and that of $\mathrm{Arg}^{446}$ render a potential interaction between the side chain and a bulky and hydrophobic +1 residue, which might be important in STAC-mediated hSIRT1 activation ${ }^{13}$. The acetyl-lysine side chain inserts into a hydrophobic cavity lined by $\mathrm{Phe}^{414}, \mathrm{Leu}^{418}$ and $\mathrm{Val}^{445}$. The acetyl group is sandwiched between $\mathrm{His}^{363}$ and $\mathrm{Phe}{ }^{297}$, with the $\varepsilon-\mathrm{N}$ of the acetyl-lysine hydrogen bonded with the carbonyl oxygen of $\mathrm{Val}^{412}$, which maintains the orientation and the extended conformation of the acetyl-lysine side chain. CarbaNAD also makes multipoint contacts with hSIRT1 (Supplementary Fig. 5b), most of which are similar to those observed in reported Sirtuin/NAD ${ }^{+}$strucutres ${ }^{19,31}$. Inhibitor 2 occupies both the acetyl-lysine-binding site and the NAM-binding C-pocket of mini-hSIRT1, similar to the recently reported structure of the SIRT3/2 complex ${ }^{30}$ (Supplementary Fig. 5c). Similar to SIRT3, binding of substrates or the active-site inhibitor leads to domain closure, bringing the small and large lobes together ${ }^{20,21,30}$. Comparison of the three structures shows that the locations of the N-terminal SBD domain relative to the catalytic core are divergent among the three structures, likely to be impacted by different crystal packings (Fig. 3c). The hinge residue, $\mathrm{Arg}^{234}$, is located within the polybasic linker (residues 233-238, KRKKRK) and anchors the N-terminal SBD to the catalytic domain through a salt bridge formed between its guanidinium group and the carboxylate group of $\mathrm{Asp}^{475}$ and hydrogen bonds to the carbonyl groups of $\mathrm{His}^{473}$ and Val ${ }^{459}$ (Supplementary Fig. 5d). In contrast, the SBD domain itself is relatively rigid, with a superimposable STAC-binding helix-turn-helix (H2-T-H3) motif with only the a

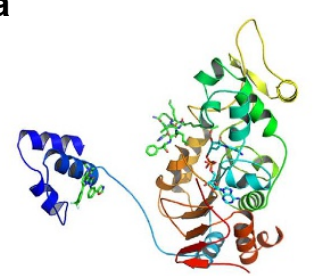

b

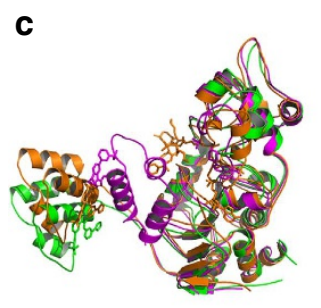

d

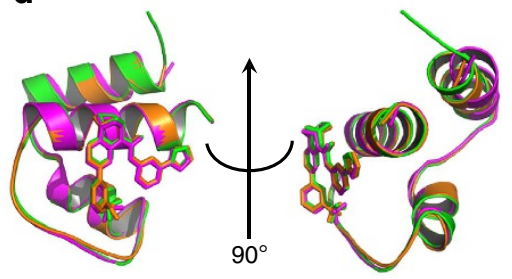

e

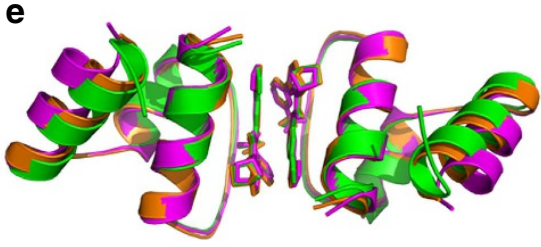

Figure 3 | Structures of Mini-hSIRT1-STAC/ligand complex. (a) Structure of mini-hSIRT1/1/Ac-p53 7-mer/CarbaNAD quaternary complex. The STAC 1 and Ac-p53 7-mer are shown in green, red and blue for carbon, oxygen and nitrogen atoms. The CarbaNAD is shown in cyan, red, blue and orange for carbon, oxygen, nitrogen and phosphate atoms. The protein ribbon is rainbow-colored from blue at the $\mathrm{N}$-terminus to red at the C-terminus. (b) Structure of mini-hSIRT1/1//2 complex. The mini-hSIRT1 shown in this complex is hSIRT1(183-516)-GS-CTR as the same complex containing hSIRT1(183-505)-(GGGS) ${ }_{2}$-CTR diffracts to $3.5 \AA$ even though the structures are almost identical. The STAC $\mathbf{1}$ is shown in green, red and blue for carbon, oxygen and nitrogen atoms. The Inhibitor $\mathbf{2}$ is shown in cyan, red, blue and yellow for carbon, oxygen, nitrogen and sulfur atoms. The protein ribbon is rainbow-colored from blue at the $\mathrm{N}$-terminus to red at the C-terminus. (c) Structural comparison of mini-hSIRT1/1 $\mathbf{1}$ complex (green), mini-hSIRT1/1/Ac-p53 7-mer/CarbaNAD quaternary complex (orange) and mini-hSIRT1/1/2 complex (magenta). (d) Superimposition of the SBD domains from mini-hSIRT1/1 complex (green), mini-hSIRT1/1/Acp53 7-mer/CarbaNAD quaternary complex (orange) and mini-hSIRT1/1/2 complex (magenta). (e) Comparison of the STAC-mediated dimer interface of mini-hSIRT1/1 complex (green), mini-hSIRT1/1/Ac-p53 7-mer/ CarbaNAD quaternary complex (orange) and mini-hSIRT1/1/2 complex (magenta).

first helix tilting out slightly in the mini-hSIRT1/1/2 complex structure (Fig. 3d). The STAC-mediated dimer interface also seems to be conserved among the three structures (Fig. 3e).

Site-directed mutagenesis of the STAC binding pocket. We used site-directed mutagenesis on the full-length hSIRT1 to confirm the key residues of the SBD that were identified by the mini-hSIRT1 structures. The following point mutants of fulllength hSIRT1 were generated probing three classes of residues: (a) residues which appear to directly interact with STACs (T219A, I223A, N226A and I227A); (b) SBD residues with no apparent role in activator binding (Q222A and V224A); and (c) $\mathrm{Glu}^{230}$ previously demonstrated to be important for SIRT1 activation $^{16}$ (E230K, E230A and E230Q) (Fig. 2b). None of the mutants significantly impaired the basal catalytic activity using the Ac-p53(W5) substrate or affected inhibition by EX-527, a 
Trifluoroacetic acid (TFA)-p53 peptide (Ac-RHK-K ${ }^{\mathrm{TFA}}$-L-Nle-F$\mathrm{NH}_{2}$ ), or NAM (Supplementary Tables 4 and 5).

The impact of the mutations on activation was first assessed by comparing the fold-activation of wild-type versus mutant fulllength SIRT1 using a structurally diverse set of 246 STACs tested at a fixed concentration of $25 \mu \mathrm{M}$. Additionally, we investigated the effect of the mutations on STAC binding versus activation by monitoring shifts in their $\mathrm{EC}_{50}$ and maximum activation values respectively using a panel of eight compounds (STACs 1, 3-9, Supplementary Fig. 2, Supplementary Tables 6 and 7). T219A, I223A and I227A all exhibit broad impairment of activation with increases in $\mathrm{EC}_{50}$ values compared with wild-type hSIRT1, suggesting impaired activator binding consistent with the mini-hSIRT1 structures (Fig. 4a and Supplementary Table 6). Interestingly, I223A was the most compound-dependent mutant, exhibiting both attenuated and enhanced activation, the latter particularly for STACs containing an ortho- $\mathrm{CF}_{3}$ substituted phenyl ring (Fig. 4a and Supplementary Fig. 6a). In the crystal structure, Ile ${ }^{223}$ lies directly beneath the STAC and lines the pocket into which the meta- $\mathrm{CF}_{3}$ of 1 inserts. The cavity created by mutation of $\mathrm{Ile}^{223}$ to Ala would be expected to better accommodate an ortho- versus a meta-substitution. This observation further validates the key molecular interactions governing STAC binding indicated in the structure and points to strategies for altering STAC interaction with the SBD.

Asn $^{26}$ appears to form a hydrogen bond between its carboxamide nitrogen and the carbonyl oxygen of $\mathbf{1}$ on the surface of the protein (Fig. 2b). However, activation of N226A was only minimally impaired compared with the wild type (Fig. 4a). The small contribution from this H-bond is likely because of its high solvent exposure.

In contrast to the above mutants, Q222A and V224A displayed normal activation which is consistent with their positions away from the STAC in the mini-hSIRT1/1 structure (Fig. $4 \mathrm{a}$ and Supplementary Fig. 6b,c). Importantly, all of these data obtained with full-length hSIRT1 are consistent with what the mini-SIRT1 crystal structures predict further validating the biochemical significance of these structures.

Despite the broad impact of the mutations described above, none of them completely abolished activation of hSIRT1 as seen with removal of the SBD. As Ile ${ }^{223}$ lies directly beneath the bound STAC and activation of I223A is highly compound-dependent, we reasoned that further mutating this residue, to incorporate a more disruptive interaction in hSIRT1, would result in a more highly activation-impaired full-length enzyme. To test this hypothesis, we prepared an I223R mutant to introduce steric bulk and charge into the hydrophobic STAC-binding site. Consistent with our hypothesis, activation is completely lost for all 246 activators using both the Ac-p53(W5) or FOXO-3a substrate peptides (Fig. $4 \mathrm{~b}$ and Supplementary Fig. $6 \mathrm{~d}$ ), while the basal catalytic activity and inhibition by EX-527, TFA-p53 peptide or NAM is not impacted in the I223R mutant (Supplementary Tables 4, 5 and 8). SEC of the STAC-binding deficient mini-hSIRT1 I223R mutant remains the same in the presence of STAC 1, confirming that the observed mini-hSIRT1 dimerization in solution is mediated by STAC 1. (Supplementary Fig. 4).

Mutation of $\mathrm{Glu}^{230}$ to either Lys or Ala has been recently reported to broadly impair activation by STACs, although the mechanism by which this occurs is unclear ${ }^{16}$. We tested activation of E230K, E230A and E230Q full-length hSIRT1 proteins and found that the maximum activation is impaired with a minimal impact on the $\mathrm{EC}_{50}$ (Supplementary Tables 6 and 7), suggesting a role for $\mathrm{Glu}^{230}$ in the formation or stabilization of the activated conformation of hSIRT1. Activation of E230Q is also broadly impaired indicating that the negative charge of

\section{a}
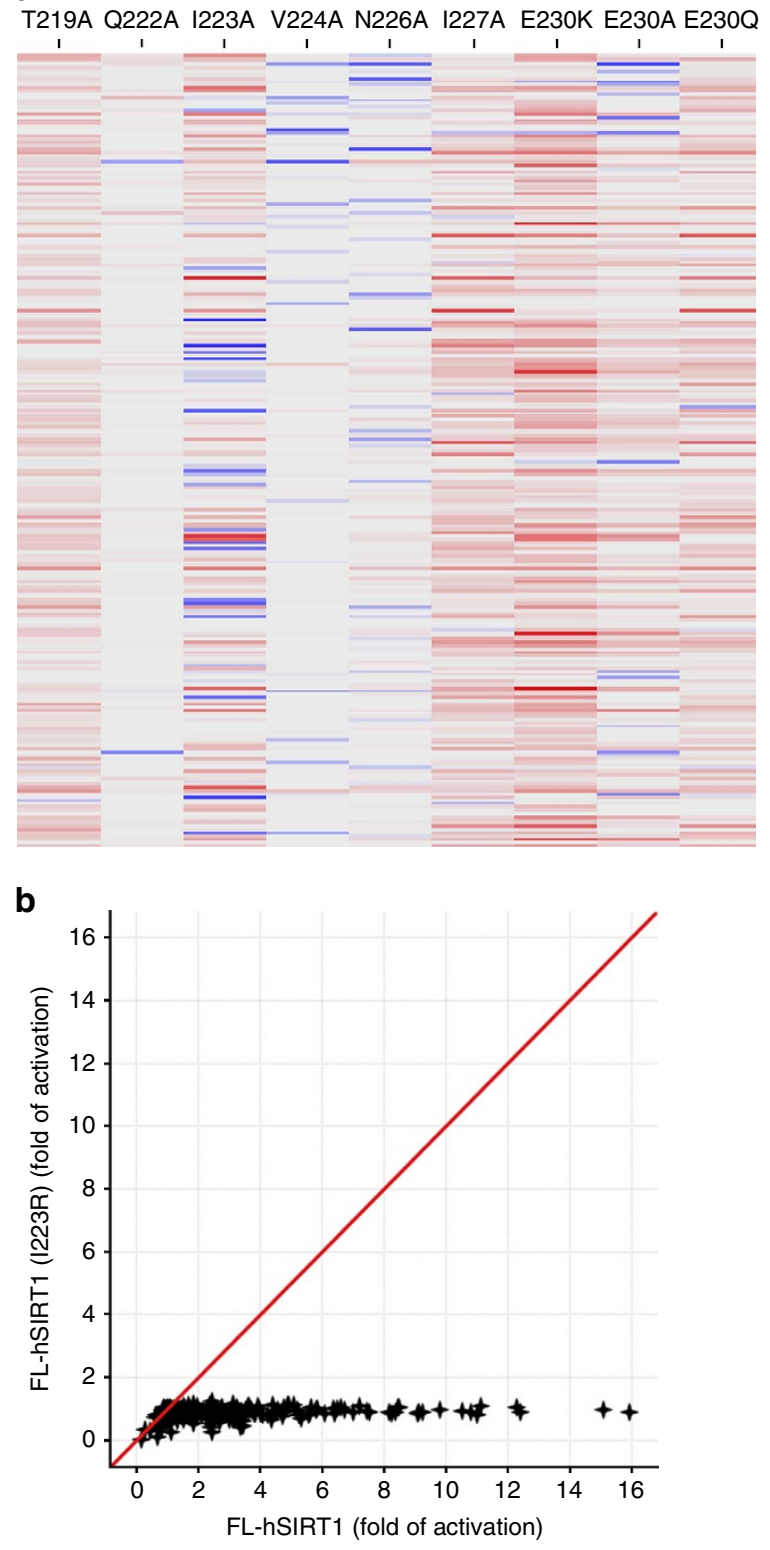

Figure 4 | Activation of full-length hSIRT1 mutants. (a) Heat map of the ratio of wild-type/mutant fold-activation for hSIRT1 mutants for each compound from a structurally diverse collection of 246 STACs. Ratios from 0.80 to 1.16 are colored gray. This range covers one s.d. of the mean for V224A (0.98 \pm 0.18-fold, Supplementary Fig. 5b) which does not affect activation. Activation impairment denoted as a red gradient (ratios of 1.17/6.78). Activation enhancement is shown as a blue gradient (ratios of $0.78 / 0.24)$. (b) Comparison of the fold-activation of I223R versus wildtype hSIRT1 with a structurally diverse collection of STACs. All of the data were generated with the OAADPr assay using the Ac-p53(W5) substrate. A different compound set ( $\sim 250$ STACs) was used for the site-directed mutagenesis studies compared with that used for the initial characterization of mini-hSIRT1 ( $~ 80$ STACs).

$\mathrm{Glu}^{230}$ is important for stabilizing the activated conformation of hSIRT1 and likely interacts with a positively charged residue in the activated state.

Allosteric coupling between STAC and substrate binding. We further probed the role of $\mathrm{Glu}^{230}$ in STAC-mediated activation using HDX-MS which revealed that, in contrast to wild-type 
hSIRT1, STAC binding to the E230K mutant no longer confers protection around the peptide-binding site in the E230K/1/Acp53(W5) complex (Fig. 5a and Supplementary Fig. 7). This indicates that the E230K mutation may negatively affect the coupling between the STAC and substrate-binding sites. The HDX-MS and activation data together suggest that Glu ${ }^{230}$ is not directly involved in STAC binding but is instead, a critical residue mediating the coupling of STAC and substrate binding to promote activation.

The observation that regions outside STAC-binding site and substrate-binding site show minimal perturbation in HDX-MS in the presence of both ligands suggests the possibility that the two binding sites might be physically close to each other in the activated conformation. Given this observation and the
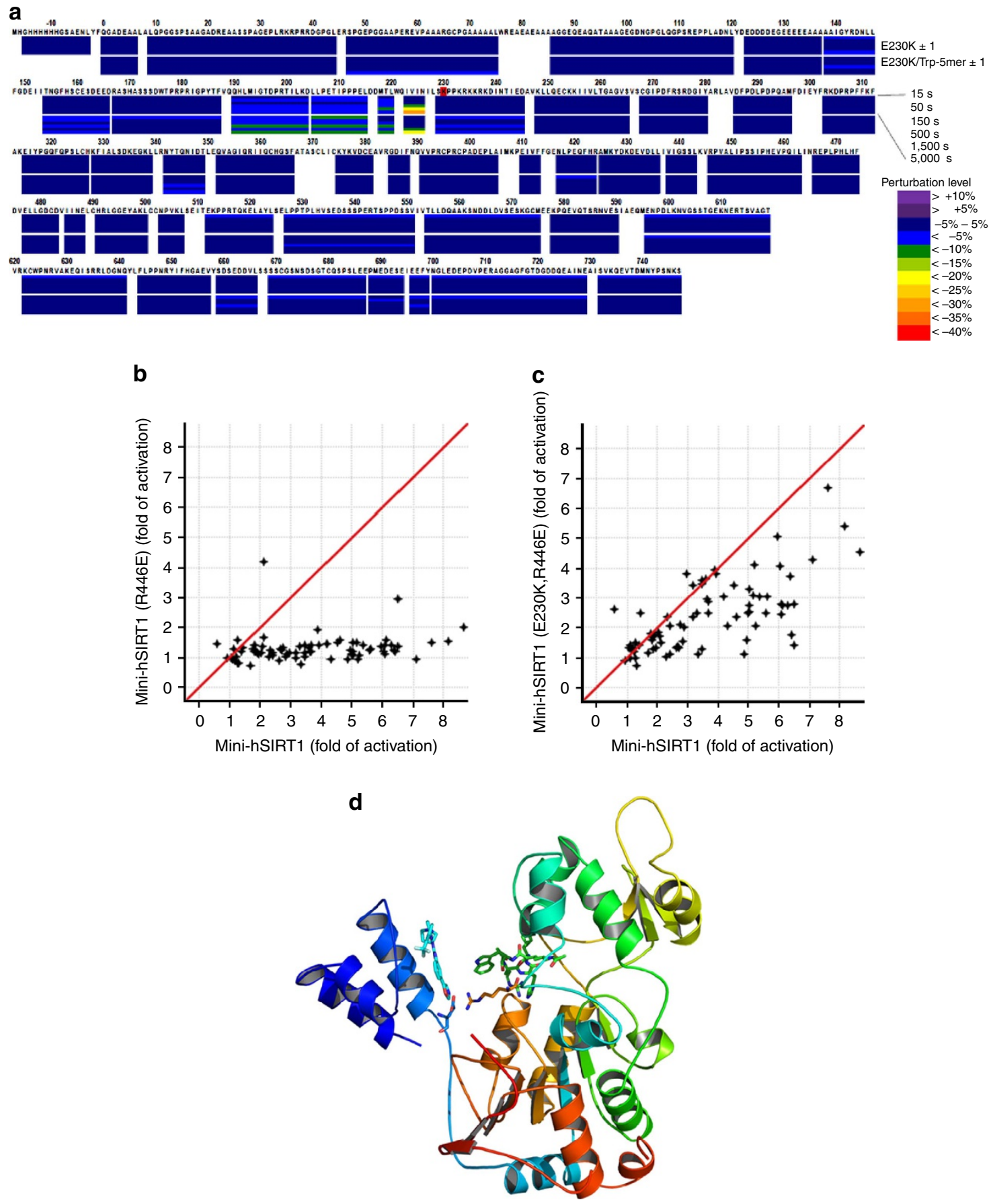

Figure 5 | E230K impairs the coupling between STAC and substrate binding and potential role of an electrostatic interaction between Glu ${ }^{230}$ and Arg $^{\mathbf{4 4 6}}$ in the activated conformation. (a) Heat map of the HDX-MS perturbation of binding of $\mathbf{1}$ to hSIRT1(E230K) in the absence or presence of Ac-p53(W5) at six different time points (15-5000 s). (b) Pivot plot of the STAC activation of mini-hSIRT1(R446E) versus mini-hSIRT1. (c) Pivot plot of the STAC activation of the double charge-reversal mutant mini-hSIRT1(R446E/E230K) versus mini-hSIRT1. (d) Speculative model of the activated conformation of SIRT1. Glu ${ }^{230}$ and $\mathrm{Arg}^{446}$ are shown in stick representation. The protein ribbon is rainbow-colored from blue at the N-terminus to red at the C-terminus. 1 and modeled Ac-p53(W5) are shown in cyan and green, respectively. 
importance of the negative charge of $\mathrm{Glu}^{230}$ for activation, we postulated that $\mathrm{Arg}^{446}$ located at the active site might be a possible electrostatic partner for $\mathrm{Glu}^{230}$, stabilizing the activated conformation of hSIRT1 and mediating the observed coupling. To this end, we made the mini-hSIRT1 R446E/E230K double mutant with E230K and R446E mini-hSIRT1 as controls. MinihSIRT1(E230K) mutant does not affect the basal catalytic activity using the Ac-p53(W5) substrate, as observed in full-length SIRT1 (Supplementary Table 2). Both mini-hSIRT1(R446E) mutant and mini-hSIRT1(E230K,R446E) mutant show higher $K_{\mathrm{M}}$ values for both peptide substrate and $\mathrm{NAD}^{+}$, which might result from the potential hydrophobic interaction between the aliphatic part of $\mathrm{Arg}^{446}$ side chain and the substrate as R446F mutant does not affect the basal catalytic activity (Supplementary Table 2). Whereas either E230K or R446E results in significant attenuation of STAC activation of mini-hSIRT1, the E230K,R446E double mutant partially restores STAC-mediated activation of minihSIRT1 compared with E230K or R446E, supporting the importance of potential electrostatic interaction between Glu ${ }^{230}$ and $\mathrm{Arg}^{446}$ in the activated conformation (Fig. 5b,c).

\section{Discussion}

In this study, we describe the design and construction of a functional mini-hSIRT1 that recapitulates three key features of full-length hSIRT1: (1) the steady-state enzyme kinetics and inhibition; (2) the STAC activation profile across multiple chemotypes; and (3) STAC activation impairment by E230K mutation. We used this mini-hSIRT1 construct to obtain the first reported crystallographic structure of hSIRT1 with a bound STAC. The biochemical and structural characterization confirms the hSIRT1 intramolecular interactions between the CTR and catalytic domain, which enhance the basal deacetylation activity of hSIRT1. The structures of the mini-hSIRT1-STAC complex reveal the detailed architecture of the STAC-binding site, which was validated in full-length hSIRT1 by site-directed mutagenesis. The STAC-binding site appears to be a shallow hydrophobic surface depression, which matches the flat and hydrophobic nature of the STACs. Consequently, the mini-hSIRT1-STAC structure reported here provides important information for future structure-based drug design. In addition, we demonstrated the coupling between the STAC-binding site and the active site using HDX-MS, which is impaired by the previously reported E230K mutant. Structure-based mutagenesis suggested that the electrostatic interaction between $\mathrm{Glu}^{230}$ and $\mathrm{Arg}^{446}$ might stabilize the activated conformation. The exact nature of the activated conformation is still elusive. Apparently, $\mathrm{Glu}^{230}$ and $\mathrm{Arg}^{446}$ in the reported structure are too far to make electrostatic interactions. Modeling with the N-terminal SBD treated as a rigid body to rotate around the hinge point $\mathrm{Arg}^{234}$ suggests that rotating the SBD around $\mathrm{Arg}^{234}$ could bring $\mathrm{Glu}^{230}$ close to Arg ${ }^{446}$, which interestingly also bring the STAC 1 close to the active site, esp. the hydrophobic side chain of the modeled Ac-p53(W5) (Fig. 5d). This model is highly speculative and needs to be tested experimentally, but does seem to be attractive as it might help to explain the requirement of some hydrophobic moiety on the peptide for SIRT1 activation by STACs, by participating in the composite activator-binding site and facilitating the formation of the activated conformation. However, some key questions remain to be answered with further investigation: (1) is the observed STAC induced SIRT1 dimer relevant for SIRT1 activation by STACs? (2) How does the STAC binding in the $\mathrm{N}$-terminal SBD enhance the substrate binding at the active site, which is not obvious from the comparison of current structures of Mini-SIRT1/1 and Mini-SIRT1/1/ p53 7-mer/CarbaNAD? (3) Does STAC binding itself induce conformational change of the N-terminal SBD, in other words, what does apo Mini-SIRT1 look like? The current structures served as a stepping stone to answer these important questions and elucidate the mechanism of activation of SIRT1 by STACs. In summary, the results presented here provide unambiguous visual and functional proof of direct allosteric activation of hSIRT1 by small molecules, and provide a basis for further elucidation of the mechanism of hSIRT1 activation by STACs.

\section{Methods}

Protein cloning, expression and purification. Mini-hSIRT1 constructs were cloned into a modified pET21b vector (Novagen). The protein was expressed in Escherichia coli BL21-Gold (DE3) cells (Stratagene) as an N-terminal fusion to a hexahistidine affinity tag with integrated Tobacco Etch Virus (TEV) protease site. A single colony was inoculated in LB media containing $100 \mu \mathrm{g} \mathrm{ml}^{-1}$ ampicillin at $37^{\circ} \mathrm{C}, 250$ r.p.m. until the $\mathrm{A}_{600}$ reached 0.3 . The culture was then transferred to $16^{\circ} \mathrm{C}$ 250 r.p.m. until the $A_{600}$ reached 0.6. Isopropyl 1-thio- $\beta$-D-galactopyranoside was added to a final concentration of $0.2 \mathrm{mM}$, and expression was continued at $16^{\circ} \mathrm{C}$, 250 r.p.m. overnight. Cells were collected by centrifugation, and the pellet was resuspended in lysis buffer ( $25 \mathrm{mM}$ HEPES, pH 7.5, $200 \mathrm{mM} \mathrm{NaCl}, 5 \%$ glycerol and $5 \mathrm{mM} 2$-mercaptoethanol) and sonicated to break the cells. Supernatant was separated from cell debris by centrifugation at $10,000 \mathrm{~g}$ for $40 \mathrm{~min}$ at $4{ }^{\circ} \mathrm{C}$ and loaded onto a Ni-NTA column (Qiagen) that equilibrated with the buffer containing $25 \mathrm{mM}$ HEPES, pH 7.5, $200 \mathrm{mM} \mathrm{NaCl}, 5 \%$ glycerol, $5 \mathrm{mM}$ 2-mercaptoethanol and $20 \mathrm{mM}$ imidazole. The column was washed with five column volumes of the buffer containing $25 \mathrm{mM}$ HEPES, pH 7.5, $200 \mathrm{mM} \mathrm{NaCl}, 5 \%$ glycerol, $5 \mathrm{mM}$ 2-mercaptoethanol and $50 \mathrm{mM}$ imidazole, and eluted with the buffer containing $25 \mathrm{mM}$ HEPES, pH 7.5, $200 \mathrm{mM} \mathrm{NaCl}, 5 \%$ glycerol, $5 \mathrm{mM}$ 2-mercaptoethanol and $250 \mathrm{mM}$ imidazole. The eluted protein was dialyzed in lysis buffer and digested with TEV protease (Invitrogen) to remove the $\mathrm{N}$-terminal His tag at $4{ }^{\circ} \mathrm{C}$ overnight. The protein was loaded on a second Ni-NTA column equilibrated with lysis buffer. The untagged protein was eluted by the buffer containing $25 \mathrm{mM}$ HEPES, pH 7.5, $200 \mathrm{mM} \mathrm{NaCl}, 5 \%$ glycerol, $5 \mathrm{mM} 2$-mercaptoethanol and $5 \mathrm{mM}$ imidazole. The purified protein was dialyzed against the dialyzing buffer containing $20 \mathrm{mM}$ Tris- $\mathrm{HCl}, \mathrm{pH} 8.0,250 \mathrm{mM} \mathrm{NaCl}, 5 \%$ glycerol and $10 \mathrm{mM}$ dithiothreitol, and concentrated. The protein was further purified by a S200 column (GE Healthcare) to $95 \%$ purity as assessed by SDS-polyacrylamide gel electrophoresis analysis stained by Coomassie Brilliant Blue R-250 and concentrated to $10-15 \mathrm{mg} \mathrm{ml}^{-1}$ in the dialyzing buffer.

Full-length human SIRT1 (hSIRT1) proteins were expressed with a N-terminal $\mathrm{His}_{6}$ tag and purified as described in Hubbard et al. ${ }^{16}$ except for Q222A, and I223R SIRT1 which were purified using an ÄKTAxpress (GE Lifesciences). Each cell paste was resuspended in buffer A $(50 \mathrm{mM}$ Tris- $\mathrm{HCl} \mathrm{pH} 7.5,250 \mathrm{mM} \mathrm{NaCl}, 25 \mathrm{mM}$ imidazole and $0.1 \mathrm{mM}$ TCEP) with 1,000 U Benzonase nuclease (Sigma-Aldrich, St Louis, MO, USA) supplemented with cOmplete, EDTA-free Protease Inhibitor Cocktail Tablets (Roche) on ice. Cells were disrupted by pulse sonication with $50 \%$ on and $50 \%$ off for 12 min total at $40 \mathrm{~W}$. Insoluble debris was removed by centrifugation. Clarified supernatant was directly loaded onto a $1 \mathrm{ml}$ HisTrap FF Crude column (GE Lifesciences). After washing with buffer A, SIRT1 was eluted with buffer B (50 mM Tris- $\mathrm{HCl} \mathrm{pH} 7.5,250 \mathrm{mM} \mathrm{NaCl}, 500 \mathrm{mM}$ imidazole and $0.1 \mathrm{mM}$ TCEP). Protein was further purified by SEC in buffer C (50 mM Tris-HC pH 7.5, $300 \mathrm{mM} \mathrm{NaCl}$ and 0.1 mM TCEP) using a Hi-load Superdex 200 16/60 column (GE Lifesciences). Enzyme concentrations were determined by Bradford assay using bovine serum albumin (BSA) as a standard. Final protein purity was assessed by gel densitometry. Proteins were confirmed by liquid chromatography/ mass spectrometry. All proteins were greater than $90 \%$ pure except V224A and T219A (80\%) and E230A (85\%).

SIRT1 deacetylation reactions. SIRT1 deacetylation reactions were performed in reaction buffer (50 mM HEPES- $\mathrm{NaOH}, \mathrm{pH} 7.5,150 \mathrm{mM} \mathrm{NaCl}, 1 \mathrm{mM}$ dithiothreitol and $1 \%$ dimethylsulfoxide (DMSO)) at $25^{\circ} \mathrm{C}$ monitoring either NAM production using the continuous $\mathrm{PNC1} / \mathrm{GDH}$ coupled assay ${ }^{32}$ or $\mathrm{O}$-acetyl ADP ribose (OAcADPr) production by mass spectrometry ${ }^{16}$. Final concentrations of the PNC1/GDH coupling system components used were 20 units per ml bovine GDH (Sigma-Aldrich), $1 \mu \mathrm{M}$ yeast PNC1, $3.4 \mathrm{mM} \alpha$-ketoglutarate and $220 \mu \mathrm{M}$ NADH or NADPH. An extinction coefficient of $6.22 \mathrm{mM}^{-1} \mathrm{~cm}^{-1}$ and a pathlength of $0.81 \mathrm{~cm}$ were used to convert the absorbance at $340 \mathrm{~nm}$ to product concentration for the $150 \mu \mathrm{l}$ reactions used. Assays monitoring OAcADPr production were performed in reaction buffer with $0.05 \%$ BSA and time points were taken by quenching the deacetylation reaction with a stop solution which gave a final concentration of $1 \%$ formic acid and 5 mM NAM. Quenched reactions were diluted fivefold with 1:1 acetonitrile:methanol and spun at 5,000 $\mathrm{g}$ for $10 \mathrm{~min}$ to precipitate protein before being analyzed with an Agilent RapidFire 200 High-Throughput Mass Spectrometry System (Agilent, Wakefield, MA) coupled to an ABSciex API 4000 mass spectrometer fitted with an electrospray ionization source. The p53-based Ac-p53(W5) (Ac-RHKKAcW-NH2) and FOXO-3a 21-mer (Ac-SADDSPSQLSKAcWPGSPTSRSS-NH2) peptides were obtained from Biopeptide. Deacetylation assays used the Ac-p53(W5) substrate unless otherwise noted. 
Substrate $K_{\mathrm{M}}$ determinations were performed by varying one substrate concentration at a fixed, saturating concentration of the second substrate. SIRT1 activation and inhibition assays were run in reaction buffer with $0.05 \%$ BSA at $25^{\circ} \mathrm{C}$ and analyzed using the OAcADPr assay. Enzyme and compound were preincubated for $20 \mathrm{~min}$ before addition of substrates. For the activation screen of full-length hSIRT1, a structurally diverse set of 246 compounds was tested in duplicate at a final concentration of $25 \mu \mathrm{M}$ each. In order to be sensitive to $K_{\mathrm{M}}$-modulating activators, substrate concentrations of approximately one-tenth their $K_{M}$ values were used. The dose-dependence of eight compounds was tested and the fold-activation data were described by equation (1)

$$
\frac{v_{x}}{v_{o}}=b+\frac{R V_{\max }-b}{1+\frac{E C_{50}}{[X]_{o}}}
$$

where $v_{x} / v_{0}$ is the ratio of the reaction rate in the presence $\left(v_{x}\right)$ versus absence $\left(v_{0}\right)$ of activator $(X), R V_{\max }$ is the relative velocity at infinite activator concentration, $E C_{50}$ is the concentration of activator required to produce one-half $\mathrm{RV}_{\max }$ and $\mathrm{b}$ is the minimum value of $v_{x} / v_{0}$

SEC assay. The assays were performed with a Superdex 75 10/300 GL column (GE healthcare) injecting $100 \mu \mathrm{l}$ samples containing $10 \mu \mathrm{M}$ mini-hSIRT1 in the absence or presence of $100 \mu \mathrm{M}$ STAC, dissolved in $50 \mathrm{mM}$ HEPES-NaOH, pH 7.5, $150 \mathrm{mM}$ $\mathrm{NaCl}$ and $0.5 \mathrm{mM}$ TCEP. Binding reactions were incubated for $1 \mathrm{~h}$ at room temperature before injection into the column.

HDX-MS. On-exchange experiment of SIRT1. H/D-exchange reactions followed by pepsin digestion, desalting, high-performance liquid chromatographic separation and mass spectrometric analysis were carried out using a fully automated system, described in detail elsewhere ${ }^{22}$. Particular to this set of experiments, on-exchange reactions were initiated by mixing $20 \mu \mathrm{l}$ of a SIRT 1 stock solution $\left(0.77 \mathrm{mg} \mathrm{ml}^{-1}\right.$ SIRT1, $\pm 3.88 \mathrm{mM}$ Ac-p53(W5), $\pm 192 \mu \mathrm{M}$ ligand, in $1.9 \%$ DMSO) and $20 \mu \mathrm{l}$ of $100 \mathrm{mM}$ phosphate, $\mathrm{pH}$ read 7.0 in $\mathrm{D} 2 \mathrm{O}$. The $50 \% \mathrm{D}_{2} \mathrm{O}$ mixture was incubated at $0{ }^{\circ} \mathrm{C}$ for $15,50,150,500,1,500$ or 5,000 s. For SIRT1 (229-516), on-exchange reactions were initiated by mixing $4 \mu \mathrm{l}$ of a SIRT1 stock solution $\left(1.36 \mathrm{mg} \mathrm{ml}^{-1}\right.$ SIRT1 (229-516), $\pm 1.67 \mathrm{mM}$ CTR peptide) and $36 \mu \mathrm{l}$ of $200 \mathrm{mM}$ phosphate, $\mathrm{pH}$ read 7.0 in D2O. The $90 \% \mathrm{D} 2 \mathrm{O}$ mixture was incubated at $0^{\circ} \mathrm{C}$ for $15,50,150,500$, 1,500 , or $5,000 \mathrm{~s}$. Addition of $20 \mu \mathrm{l}$ of $1.6 \mathrm{M}$ guanidine hydrochloride $(\mathrm{GuHCl})$, $0.8 \%$ formic acid, $\mathrm{pH} 2.3$, quenched the on-exchange reaction immediately prior to being analyzed.

General protein process for standard HDX sample. The quenched solution was passed through a pepsin column $(104 \mu \mathrm{l}$ bed volume) filled with porcine pepsin (Sigma) immobilized on Poros 20 AL media (Life Technologies, Carlsbad, CA

USA) per the manufacturer's instructions, with $0.05 \%$ aqueous TFA

$\left(200 \mu \mathrm{lmin}^{-1}\right)$ for $2 \mathrm{~min}$. The digested fragments were temporarily collected onto a reverse phase trap column ( $4 \mu \mathrm{l}$ bed volume) and desalted. The peptide fragments were then eluted from the trap column and separated by a C18 column (BioBacis18; Thermo Scientific, San Jose, CA, USA) with a linear gradient of $13 \%$ solvent B to $40 \%$ solvent B over 23 min (solvent A, $0.05 \%$ TFA in water; solvent B, $95 \%$

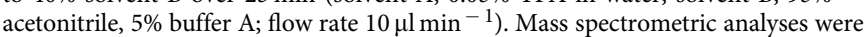

carried out using a LTQ OrbiTrap XL mass spectrometer (Thermo Fisher Scientific) with capillary temperature at $200^{\circ} \mathrm{C}$.

Digestion/separation optimization and nondeuterated experiment of SIRT1. Before H/D-exchange experiment, digestion and separation conditions were optimized to yield high sequence coverage of SIRT1 by peptic fragments with high resolution under nondeuterated conditions. In this step, a mixture of $20 \mu \mathrm{l}$ of $0.77 \mathrm{mg} \mathrm{ml}^{-1}(9.2 \mu \mathrm{M})$ SIRT1 and $20 \mu \mathrm{l}$ of $\mathrm{H} 2 \mathrm{O}$ was quenched by the addition of $20 \mu \mathrm{l}$ of various acidic buffers. For SIRT1 (229-516), a mixture of $4 \mu \mathrm{l}$ of a SIRT1 stock solution (1.36 $\mathrm{mg} \mathrm{ml}^{-1}$ SIRT1 (229-516) and $\pm 1.67 \mathrm{mM}$ CTR peptide) and $36 \mu \mathrm{l}$ of $\mathrm{H} 2 \mathrm{O}$ was quenched by the addition of $20 \mu \mathrm{l}$ of various acidic buffers. The quenched mixtures were subjected to aforementioned general protein process. The nondeuterated peptic fragments were identified by Sequest in Proteome Discoverer 1.1 (Thermo Fisher Scientific).

Fully deuterated experiment of SIRT1. The fully deuterated sample was prepared by incubating a mixture of $45 \mu \mathrm{l}$ of $0.77 \mathrm{mg} \mathrm{ml}^{-1}(9.2 \mu \mathrm{M})$ SIRT1 with $45 \mu \mathrm{l}$ of $100 \mathrm{mM}$ TCEP in D2O, pH 2.5 at $60^{\circ} \mathrm{C}$ for $3 \mathrm{~h}$. For SIRT1 (229-516), the fully deuterated sample was prepared by incubating a mixture of $9 \mu \mathrm{l}$ of $1.36 \mathrm{mg} \mathrm{ml}^{-1}(41.7 \mu \mathrm{M})$ SIRT1 $(229-516)$ with $81 \mu \mathrm{l}$ of $100 \mathrm{mM}$ TCEP in D2O, $\mathrm{pH} 2.5$ at $60^{\circ} \mathrm{C}$ for $3 \mathrm{~h}$. After incubation, the sample was kept at $0^{\circ} \mathrm{C}$ before being quenched identically to an on-exchanged solution and subjected to the general protein process.

Determination of deuteration level of each peptide after on-exchange reaction.

The centroids of peptide isotopic envelopes were measured using the in-houseprogram developed in collaboration with Sierra Analytics (Modesto, CA, USA) Corrections for back-exchange during the protein processing step were made employing the following standard equation equation (2):

$$
\text { Deuteration level }(\%)=\frac{m(P)-m(N)}{m(F)-m(N)} \times 100
$$

where $m(P), m(N)$ and $m(F)$ are the centroid value of partially deuterated (onexchanged) peptide, nondeuterated peptide and fully deuterated peptide, respectively.

Protein crystallization, data collection and structure determination. The crystals of mini-hSIRT1/1 binary complex were obtained by hanging drop vapor diffusion method at $18^{\circ} \mathrm{C}$. The crystals appeared overnight and grew to a final size of $\sim 0.1 \times 0.1 \times 0.1 \mathrm{~mm}$ within 2 days. $10 \mathrm{mg} \mathrm{ml}^{-1}$ protein was incubated with compound 1 for $\sim 1 \mathrm{~h}$ and the molar ratio of compound 1:protein is $5: 1$ with $1 \%$ DMSO. The drop was composed of $1 \mu \mathrm{l}$ of protein/compound mixture and $1 \mu \mathrm{l}$ crystallization buffer of $0.2 \mathrm{M}$ Magnesium chloride, $0.1 \mathrm{M}$ Tris $\mathrm{pH} 8.5$, and $16 \% \mathrm{w} / \mathrm{v}$ PEG 4000. The crystals of mini-hSIRT1/1/2 were obtained by hanging drop vapor diffusion method at $18^{\circ} \mathrm{C}$. The crystals appeared overnight and grew to a final size of $\sim 0.1 \times 0.1 \times 0.1 \mathrm{~mm}$ within 2 days. $10 \mathrm{mg} \mathrm{ml}^{-1}$ protein was incubated with compound 1 for about $1 \mathrm{~h}$, then incubated with compound 2 for $2 \mathrm{~h}$ and the molar ratio of compound 1:compound 2:protein is 5:5:1 with 2\% DMSO. The drop was composed of $1 \mu \mathrm{l}$ of protein/compound mixture and $1 \mu \mathrm{l}$ crystallization buffer of 0.55 M Sodium chloride, 0.1 M MES pH 6.5 and 20\% w/v PEG 4000. The crystals of mini-hSIRT1/1/p53-7mer/carbaNAD complex were obtained by hanging drop vapor diffusion method at $18^{\circ} \mathrm{C}$. The crystals appeared overnight and grew to a

Table 1 | Data processing and refinement statistics.

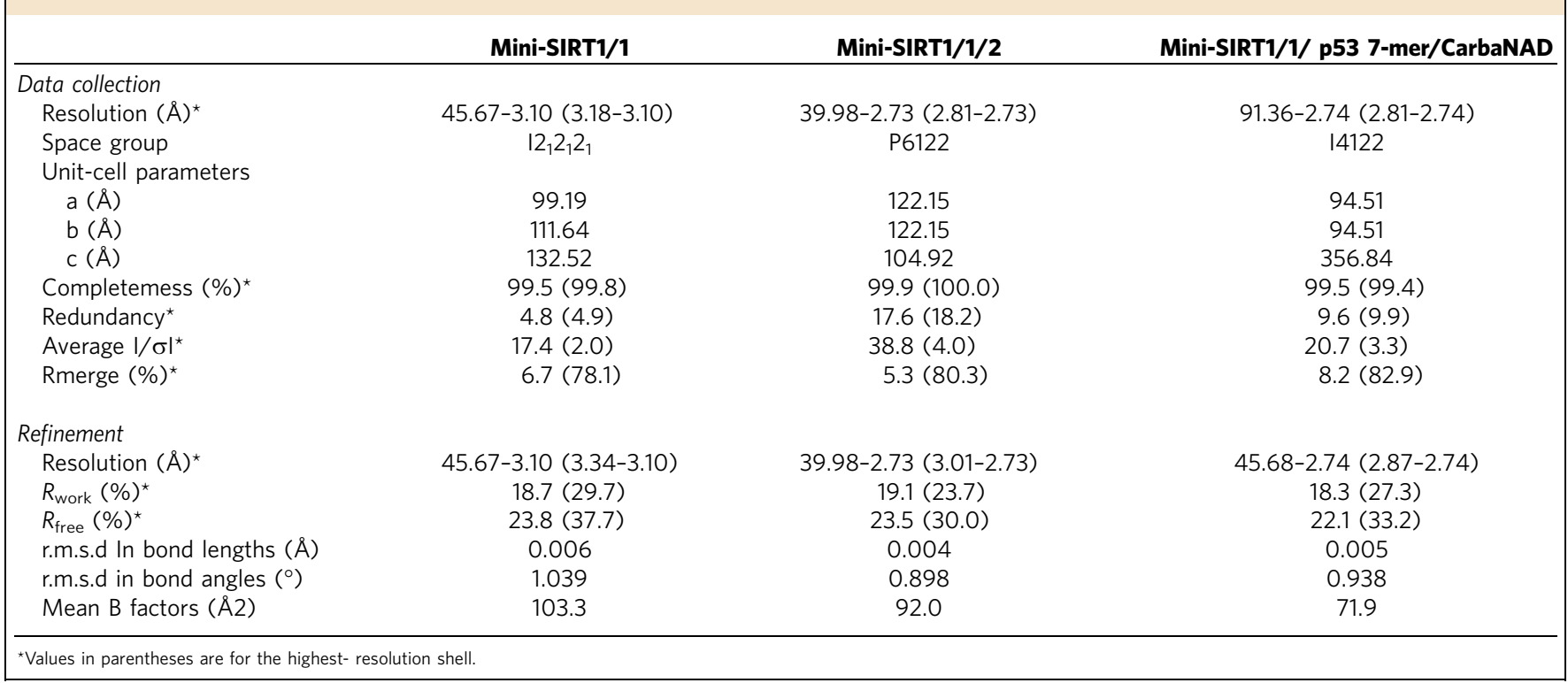


final size of $\sim 0.1 \times 0.1 \times 0.1 \mathrm{~mm}$ within 2 days. $10 \mathrm{mg} / \mathrm{ml}$ protein was incubated with compound $\mathbf{1}$ for about $1 \mathrm{~h}$, then incubated with p53-7mer and CarbaNAD for $2 \mathrm{~h}$ and the molar ratio of compound 1:p53-7mer: CarbaNAD:protein is 5:5:10:1 with $1 \%$ DMSO. The drop was composed of $1 \mu \mathrm{l}$ of the protein/compound/substrate mixture and $1 \mu \mathrm{l}$ of the crystallization buffer of $5 \% \mathrm{v} / \mathrm{v}$ Tacsimate, pH.00.1 M HEPES pH 7.0 and 10\% w/v PEG 5000 MME

The crystals were cryo-protected in mother liquor containing $20 \%$ glycerol before being flash-frozen in liquid nitrogen. Diffraction data were collected at SSRF BL17U1, APS 21-ID-D or APS 21-ID-G beamlines at $100 \mathrm{~K}$ and processed using the Xia2 program ${ }^{33}$. The molecular replacement software Phaser ${ }^{34}$ was used to solve the structure with a search model containing residues 242-494 based on the homolog model of SIRT3 (PDB code: 3GLU) initially and later also with a search model of SIRT1 (PDB code 4IG9) when available. Iterative structure refinement and model building were performed between Phenix.refine $e^{35}$ and $\operatorname{Coot}^{36}$. Bulk solvent correction and Translation/Libration/Screw-motion (TLS) refinement were used during the refinement and model building. Detailed information regarding the diffraction data, refinement and structure statistics is listed in Table 1.

Details of the chemical compounds synthesismethods are provided in Supplementary Methods.

\section{References}

1. Finkel, T., Deng, C. X. \& Mostoslavsky, R. Recent progress in the biology and physiology of sirtuins. Nature 460, 587-591 (2009).

2. Baur, J. A., Ungvari, Z., Minor, R. K., Le Couteur, D. G. \& de Cabo, R. Are sirtuins viable targets for improving healthspan and lifespan? Nat. Rev. Drug. Discov. 11, 443-461 (2012).

3. Frye, R. A. Phylogenetic classification of prokaryotic and eukaryotic Sir2-like proteins. Biochem. Biophys. Res. Commun. 273, 793-798 (2000).

4. Haigis, M. C. \& Sinclair, D. A. Mammalian sirtuins: biological insights and disease relevance. Аnnu. Rev. Pathol. 5, 253-295 (2010).

5. Verdin, E., Hirschey, M. D., Finley, L. W. \& Haigis, M. C. Sirtuin regulation of mitochondria: energy production, apoptosis, and signaling. Trends. Biochem. Sci. 35, 669-675 (2010)

6. Haigis, M. C. \& Guarente, L. P. Mammalian sirtuins-emerging roles in physiology, aging, and calorie restriction. Genes Dev. 20, 2913-2921 (2006).

7. Sebastian, C., Satterstrom, F. K., Haigis, M. C. \& Mostoslavsky, R. From sirtuin biology to human diseases: an update. J. Biol. Chem. 287, 42444-42452 (2012).

8. Banks, A. S. et al. SirT1 gain of function increases energy efficiency and prevents diabetes in mice. Cell. Metab. 8, 333-341 (2008).

9. Kim, D. et al. SIRT1 deacetylase protects against neurodegeneration in models for Alzheimer's disease and amyotrophic lateral sclerosis. $E M B O$. J. 26, 3169-3179 (2007).

10. Yoshizaki, T. et al. SIRT1 inhibits inflammatory pathways in macrophages and modulates insulin sensitivity. Am. J. Physiol. Endocrinol. Metab. 298, E419-E428 (2009).

11. Yoshizaki, T. et al. SIRT1 exerts anti-inflammatory effects and improves insulin sensitivity in adipocytes. Mol. Cell. Biol. 29, 1363-1374 (2009).

12. Milne, J. C. et al. Small molecule activators of SIRT1 as therapeutics for the treatment of type 2 diabetes. Nature 450, 712-716 (2007)

13. Dai, H. et al. SIRT1 activation by small molecules: kinetic and biophysical evidence for direct interaction of enzyme and activator. J. Biol. Chem. 285, 32695-32703 (2010)

14. Howitz, K. T. et al. Small molecule activators of sirtuins extend Saccharomyces cerevisiae lifespan. Nature 425, 191-196 (2003).

15. Pacholec, M. et al. SRT1720, SRT2183, SRT1460, and resveratrol are not direct activators of SIRT1. J. Biol. Chem. 285, 8340-8351 (2010).

16. Hubbard, B. P. et al. Evidence for a common mechanism of SIRT1 regulation by allosteric activators. Science 339, 1216-1219 (2013).

17. Zhao, X. et al. The 2.5A crystal structure of the sirt1 catalytic domain bound to nicotinamide adenine dinucleotide $(\mathrm{NAD}(+))$ and an indole (EX527 analogue) reveals a novel mechanism of histone deacetylase inhibition. J. Med. Chem. 56, 963-969 (2013)

18. Davenport, A. M., Huber, F. M. \& Hoelz, A. Structural and functional analysis of human SIRT1. J. Mol. Biol. 426, 526-541 (2014).

19. Yuan, H. \& Marmorstein, R. Structural basis for sirtuin activity and inhibition. J. Biol. Chem. 287, 42428-42435 (2012)

20. Jin, L. et al. Crystal structures of human SIRT3 displaying substrate-induced conformational changes. J. Biol. Chem. 284, 24394-24405 (2009).

21. Szczepankiewicz, B. G. et al. Synthesis of carba-NAD and the structures of its ternary complexes with SIRT3 and SIRT5. J. Org. Chem. 77, 7319-7329 (2012).

22. Hamuro, Y. et al. Rapid analysis of protein structure and dynamics by hydrogen/ deuterium exchange mass spectrometry. J. Biomol. Tech. 14, 171-182 (2003).

23. Kang, H. et al. Peptide switch is essential for Sirt1 deacetylase activity. Mol. Cell. 44, 203-213 (2011).

24. Pan, M., Yuan, H., Brent, M., Ding, E. C. \& Marmorstein, R. SIRT1 contains $\mathrm{N}$ - and C-terminal regions that potentiate deacetylase activity. J. Biol. Chem. 287, 2468-2476 (2012).
25. Robinson, C. R. \& Sauer, R. T. Optimizing the stability of single-chain proteins by linker length and composition mutagenesis. Proc. Natl. Acad. Sci. USA 95, 5929-5934 (1998).

26. Napper, A. D. et al. Discovery of indoles as potent and selective inhibitors of the deacetylase SIRT1. J. Med. Chem. 48, 8045-8054 (2005).

27. Lakshminarasimhan, M. et al. Molecular architecture of the human protein deacetylase Sirt1 and its regulation by AROS and resveratrol. Biosci. Rep. 33, 395-404 (2013).

28. Vu, C. B. et al. Discovery of imidazo[1,2-b]thiazole derivatives as novel SIRT1 activators. J. Med. Chem. 52, 1275-1283 (2009).

29. Hsu, H. C. et al. (2013) Structural basis for allosteric stimulation of Sir2 activity by Sir4 binding. Genes Dev. 27, 64-73 (2013).

30. Disch, J. S. et al. Discovery of thieno[3,2-d]pyrimidine-6-carboxamides as potent inhibitors of SIRT1, SIRT2, and SIRT3. J. Med. Chem. 56, 3666-3679 (2013).

31. Sanders, B. D., Jackson, B. \& Marmorstein, R. Structural basis for sirtuin function: what we know and what we don't. Biochim. Biophys. Acta 1804, 1604-1616 (2010).

32. Smith, B. C., Hallows, W. C. \& Denu, J. M. A continuous microplate assay for sirtuins and nicotinamide-producing enzymes. Anal. Biochem. 394, 101-109 (2009).

33. Winter, G. xia2: An expert system for macromolecular crystallography data reduction. J. Appl. Crystallogr. 43, 186-190 (2010).

34. McCoy, A. J. et al. Phaser crystallographic software. J. Appl. Crystallogr. 40, 658-674 (2007).

35. Adams, P. D. et al. PHENIX: building new software for automated crystallographic structure determination. Acta Crystallogr. D Biol. Crystallogr. 58, 1948-1954 (2002).

36. Emsley, P. \& Cowtan, K. Coot: model-building tools for molecular graphics. Acta Crystallogr. D Biol. Crystallogr. 60, 2126-2132 (2004).

\section{Acknowledgements}

We thank Meidy Lontoh, Sara Grab and Eli Schuman for compound plating and logistics. We are grateful for supervision of protein production and X-ray diffraction data collection by Derek Ren, Wentao Wei and Jianhua Cai at Viva Biotech. We thank Matthew Lochansky for HRMS analysis and Karen A. Evans, Minghui Wang and Yanqiu Qian for NMR analysis. The authors also thank Vipin Suri, Christine Loh, Nino Campobasso, Kevin Madauss, Marti Head, Andrew Maynard, William H. Miller, David A. Sinclair and Leonard P. Guarente for helpful discussion.

\section{Author contributions}

H.D.,D.Q.,and F.J. contributed to the structural biology experiments. H.D., A.W.C., T.V.R., T.C., B.P. and F.B. contributed to the enzymology experiments. H.D., J.E. and Y.H. contributed to the HDX-MS experiments. H.Z., Y.J., D.Q., B.W. and J.W. contributed to the protein expression, purification and characterization. C.A.B., J.S.D, R.C., B.S., C.O., P.Y.N., B.H.W., R.C., R.N., K.K. and M.W. contributed to the preparation and characterization of the final compounds or intermediates. H.D., T.V.R., S.M.S and B.S. designed the project. H.D., T.V.R., S.M.S, B.S., C.M., E.H., J.C.W., R.B.P., G.P.V. and J.L.E. supervised the project. H.D., T.V.R. J.C.W., R.B.P., G.P.V., and J.L.E. wrote the manuscript. All authors critically read and contributed to the manuscript.

\section{Additional information}

Accession codes. The atomic coordinates of the crystal structures of the mini-hSIRT1/1 complex, the mini-hSIRT1/1/2 complex, and the mini-hSIRT1/1/p53-7mer/carbaNAD complex have been deposited in the Protein Data Bank (accession codes $4 \mathrm{ZZH}, 4 \mathrm{ZZI}$ and4ZZJ).

Supplementary Information accompanies this paper at http://www.nature.com/ naturecommunications

Competing financial interests: H.D., A.W.C., T.V.R., T.C., H.Z., Y.J., S.M.S., B.P., B.S. C.A.B., J.S.D., R.C., B.S., C.O., P.Y.N., B.H.W., R.C., R.N., K.K., F.B., M.W., E.H., J.C.W. R.B.P., G.P.V. and J.L.E. are employees GlaxoSmithKline. Patent applications relating to the synthetic compounds have been filed.

Reprints and permission information is available online at http://npg.nature.com/ reprintsandpermissions/

How to cite this article: Dai, H. et al. Crystallographic structure of a small molecule SIRT1 activator-enzyme complex. Nat. Commun. 6:7645 doi: 10.1038/ncomms8645 (2015)

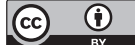

This work is licensed under a Creative Commons Attribution 4.0 International License. The images or other third party material in this article are included in the article's Creative Commons license, unless indicated otherwise in the credit line; if the material is not included under the Creative Commons license, users will need to obtain permission from the license holder to reproduce the material To view a copy of this license, visit http://creativecommons.org/licenses/by/4.0/ 